\title{
Homocysteine and Parkinson's Disease: A Complex Relationship
}

\section{Gayle Helane Doherty*}

School of Psychology and Neuroscience, University of St Andrews, Scotland, UK

\begin{abstract}
The scientific literature contains a multitude of papers linking homocysteine to poor health. Elevated homocysteine levels have been linked to cardiovascular disorders, an increased risk of fractures in the elderly and to neurological conditions. Parkinson's Disease is the second most prevalent neurodegenerative disorder after Alzheimer's disease. It is characterised primarily by the loss of dopaminergic neurons of the substantia nigra pars compacta leading to movement disorders. In vitro data has demonstrated a marked deleterious effect of homocysteine on the neurons that die in Parkinson's. Yet population-based studies reveal mixed results as to whether or not elevated homocysteine is a risk factor for Parkinson's development. Nonetheless it is well known that the use of levodopa to alleviate symptoms of Parkinson's leads to hyperhomocysteinemia, and that patients with the highest levels of homocysteine deteriorate faster than those with lower homocysteine titres. Thus homocysteine is of clinical significance for Parkinson's patients and a fuller understanding of its effects on neuronal biochemistry and function will lead to better strategies to maintain quality of life for Parkinson's sufferers.
\end{abstract}

Keywords: DNA methylation; Homocysteine; Levodopa; Neurodegeneration; Nitrosylation; Parkinson's Disease

Abbreviations: ALS: Amyotrophic Lateral Sclerosis; ATP: Adenosine Triphosphate; CBS: Cystathionine $\beta$-Synthase; CNS: Central Nervous System; COMT: Catechol-O-Methyl-Transferase; CYP2E1: Cytochrome P450 2E1; eNOS: Endothelial Nitric Oxide Synthase; Hcy: Homocysteine; L-DOPA: Levodopa; mPT: Mitochondrial Permeability Transition; MPTP: 1-Methyl 4-Phenyl 1,2,3,6-Tetrahydropyridine; MTHFR: Methylene Tetrahydrofolate Reductase; NMDA: $N$-methyl-D-Aspartate; NO: Nitric Oxide; 6-OHDA: 6-Hydroxydopamine; PD: Parkinson's Disease; PON1: Paraoxonase 1; ROS: Reactive Oxygen Species; SAH: S-Adenosyl-Hcy; SAM: S-Adenosyl Methionine; UPDRS: Unified Parkinson's Disease Rating Scale; VNTR: Variable Number Tandem Repeat

\section{Introduction}

Homocysteine $(\mathrm{Hcy})$ is a sulphur-containing non-protein amino acid that is a naturally-occurring by-product of the S-adenosyl methionine (SAM) cycle. Two pathways are employed by the body to maintain Hcy levels within a narrow concentration range leaving sufficient Hcy to contribute to cellular biochemical pathways yet preventing it from building up to concentrations that can be deleterious to health. The definition of normal levels of plasma $\mathrm{Hcy}$ varies. However, it is widely accepted that normal plasma Hcy levels range from 5-15 $\mu \mathrm{M}$; moderate hyperhomocysteinemia 15$30 \mu \mathrm{M}$; intermediate hyperhomocysteinemia $30-100 \mu \mathrm{M}$; severe hyperhomocysteinemia $>100 \mu \mathrm{M}$ [1]; and values of 200-300 $\mu \mathrm{M}$ have been recorded in some homocystinuric patients [2]. Numerous studies and reviews have considered the hypothesis that elevated Hcy levels impact negatively on cellular health and constitute a risk factor for a variety of conditions including neurodegeneration.

Parkinson's disease (PD) is the second most prevalent neurodegenerative condition after Alzheimer's disease [3]. It is a progressive neurological disorder characterized by loss of neurons including those of the substantia nigra pars compacta, with consequent dopamine depletion from the striatum leading to motor control problems [4]. The cellular pathology of PD reveals degeneration of primarily dopaminergic neurons, the presence of eosinophilic intracytoplasmic Lewy bodies, dystrophic neurites, mitochondrial dysfunction and neuroinflammation [5,6]. Medical advances and changing lifestyles have led to a continuing global demographic shift towards an ageing population. In 2001, the UK census revealed for the first time, a greater number of over 65 s than under 16s [7]. Sadly, ill-health occurs more frequently in the over 65 s than in younger age groups, impacting on the economy, society and most strongly on the friends and carers of the elderly [8]. Accordingly, neurodegenerative conditions such as PD are more prevalent in the elderly. Thus whilst PD can afflict very young individuals with juvenile onset occurring before 20 years of age and early onset before 40 years of age [9], ageing is widely acknowledged as the greatest risk factor for the development of the condition [10].

Elevated levels of Hcy in a cross sectional study of Parkinson's patients compared to age-matched controls were first reported in the literature nearly twenty years ago [11]. However, data suggesting that elevated Hcy levels are a risk factor for the development of PD are lacking. As such, a number of studies have indicated that individuals who carry genetic mutations that cause a mild elevation in Hcy levels are at no greater risk of PD than individuals lacking these alleles. Nonetheless, Hcy levels are shown to directly correlate with the rate of PD progression suggesting a potentially important role once the disease has been established. This review will consider the complicated relationship between Hcy and PD, with reference to pertinent molecular and cellular pathways that are central to PD.

\section{The SAM Cycle and Hcy Production}

Hcy has a number of essential functions within the cell when present within a normal physiological range. In mammals, Hcy is required for cysteine biosynthesis. It can also maintain methionine levels via the SAM cycle. Hcy concentrations are usually maintained

*Corresponding author: Gayle Helane Doherty, School of Psychology and Neuroscience, University of St Andrews, Bute Building, West Burn Lane, St Andrews, Fife, KY16 9TS, UK, Tel: +44 1334 463611; E-mail: ghm@st-andrews.ac.uk

Received February 20, 2013; Accepted March 22, 2013; Published March 25, 2013

Citation: Doherty GH (2013) Homocysteine and Parkinson's Disease: A Complex

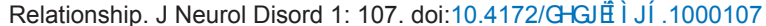

Copyright: (c) 2013 Doherty GH. This is an open-access article distributed under the terms of the Creative Commons Attribution License, which permits unrestricted use, distribution, and reproduction in any medium, provided the original author and source are credited. 
in the sub-toxic range due the actions of the pathways that lead to methionine and cysteine biosynthesis, the transmethylation and transsulfuration pathways respectively (Figure 1).

In the transmethylation pathway, Hcy's role lies at the conjunction of two metabolic cycles: the SAM cycle and the folate cycle $[1,12]$. The SAM cycle begins with influx of methionine from the diet, which reacts with adenosine triphosphate (ATP), to form SAM [13]. This reaction requires methionine methyltransferase as a catalyst. SAM has a number of important biological functions. It can be decarboxylated to form $\mathrm{S}$-adenosylmethioninamine, which then donates an $\mathrm{n}$-propylamine as part of polyamine biosynthesis. Polyamines per se have a number of important roles including enhancing the permeability of the blood brain barrier [14] and modulating ion channels that are known to be important for neuronal function such as the N-methyl-D-aspartate (NMDA) receptor [15]. In addition, SAM acts as a methyl donor in a wide range of transmethylation reactions and can be cleaved by a number of enzymes, termed radical SAM enzymes, producing a radical as an intermediate [16]. In the SAM cycle, SAM-dependent methylases convert SAM to S-adenosyl homocysteine (SAH) [17], which is broken down to produce Hcy and adenosine through the activity S-adenosyl homocysteine hydrolase. At this point, Hcy has two possible fates. It can either be re-methylated to form methionine or broken down via the transsulfuration pathway.

Re-methylation of Hcy to methionine requires a methyl donor. Choline, part of the B vitamin complex, is a direct precursor of betaine. This, in turn, is a methyl donor that converts Hcy to methionine and dimethylglycine [18]. Furthermore, methyl tetrahydrofolate can act as a methyl donor to Hcy triggering the folate cycle. This reaction requires methionine synthase as a catalyst, together with vitamin B12 as a co-factor. Thus re-methylation of Hcy gives rise to methionine and tetrahydrofolate. Serine hydroxy-methyl transferase

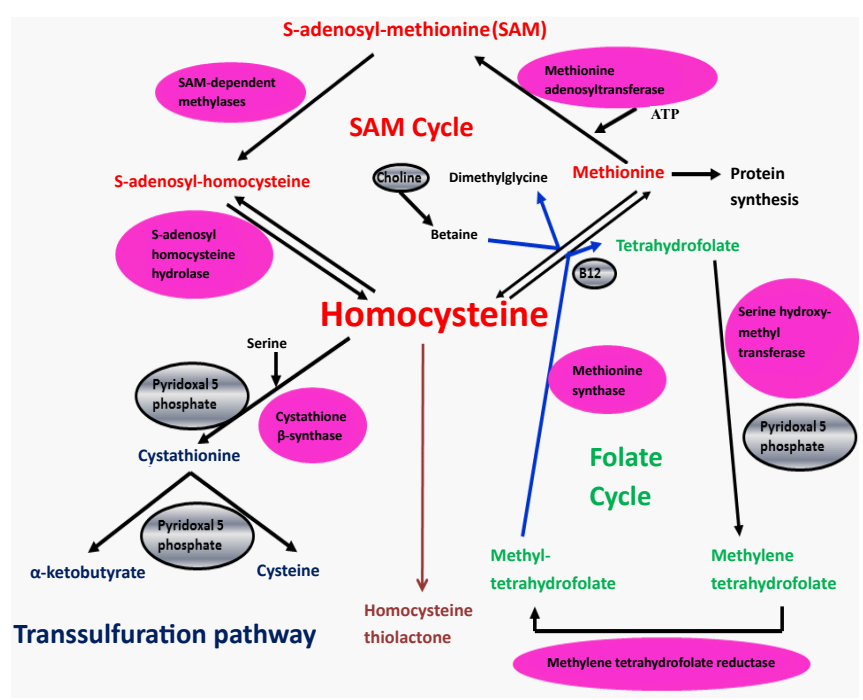

Figure 1: Biochemistry of Hcy Metabolism

Homocysteine is metabolised via two pathways: the S-adenosyl methionine (SAM) cycle (shown in red), which re- methylates Hcy back to methionine via the folate cycle (shown in green); and the transsulfuration pathway (shown in purple), which breaks down excess Hcy in preparation for excretion from the body. Methylation reactions are highlighted by blue arrows. Members of the B vitamin family, deficiencies in which promote hyperhomocysteinemia, are highlighted in grey oval boxes. Critical enzymes in these pathways are shown in pink ovals. In addition to these pathways, homocysteine can be biochemically altered within the cell to form homocysteine thiolactone (maroon). interacts with tetrahydrofolate, in a reaction that requires pyridoxal 5-phosphate (active vitamin B6) as a co-factor, to produce methylene tetrahydrofolate and glycine. Thereafter, methylene tetrahydrofolate is reduced to methyl tetrahydrofolate completing the folate cycle [19] (Figure 1).

In the transsulfuration pathway, Hcy condenses with serine to form cystathionine utilising cystathionine $\beta$-synthase (CBS) as a catalyst for this interaction. This also requires the presence of pyridoxal 5 -phosphate as a co-factor [20]. Cystathionine $\gamma$-lyase hydrolyses the cystathionine that is produced to form the non-essential amino acid cysteine and $\alpha$-ketobutyrate $[21,22]$. Cystathionine $\gamma$-lyase activity is more than 100 fold lower in the brain than in the liver [23], although the transsulfuration pathway is active in the central nervous system (CNS) [24]. To date it is unknown whether the lower activity of cystathionine $\gamma$-lyase in the CNS makes it harder for Hcy levels to be moderated within the brain. In addition to the above pathways, Hcy can be further metabolised within the cell, for instance by formation of the cyclic thioester Hcy thiolactone, which, as will be discussed later, can cause protein damage and dysfunction.

\section{Hyperhomocysteinemia: Causes and Treatments}

Elevated plasma Hcy can result from genetic or acquired factors, or an interaction between the two. The genetic pathways underpinning extreme elevations in Hcy have been known for over half a century. Patients with severe hyperhomocysteinemia or homocystinuria, a multi-system metabolic disorder characterised by the presence of excess Hcy in the urine, demonstrate homozygous inheritance of null mutations in either the cystathionine $\beta$-synthase (CBS) gene or the methylene tetrahydrofolate reductase (MTHFR) gene. These null mutations result in CBS deficiency, preventing the transsulfuration pathway from metabolising Hcy to cystathionine, or in MTHFR deficiency, inhibiting remethylation of Hcy to methionine via the folate cycle, respectively $[25,26]$.

Based on the above findings it can be postulated that heterozygositiy for CBS or MTHFR null mutant alleles could lead to more moderate elevations in Hcy. Thus, individuals who are heterozygous for a null mutation in the CBS gene have higher plasma levels of Hcy than individuals not carrying this allele [27]. Heterozygosity for the C677T mutation in the MTHFR gene, leads to elevated plasma Hcy only when dietary folate supply is low [28], therefore dietary factors are crucial in determining whether or not heterozygosity leads to elevated plasma Hcy per se.

Hyperhomocysteinemia can also occur due to mutations in genes that are not linked to the SAM cycle. For example, the intron $4 \mathrm{a} 4 \mathrm{~b}$ variable number tandem repeat (VNTR) polymorphism in the endothelial nitric oxide synthase (eNOS) gene that synthesises nitric oxide (NO) is linked to elevated total plasma Hcy in males [29]. It has been shown that polymorphisms in intron 4 of eNOS are associated with enhanced NO production [30]. The potential mechanisms whereby alterations in eNOS may induce an increase in Hcy include NO-mediated inhibition of methionine synthase [31] and NO inhibiting the synthesis of ferritin that is required for oxidative cleavage of folate $[32,33]$. However, more data is required to clarify precisely how this polymorphism leads to elevated Hcy levels in patients. There are further pathways that can lead to increased Hcylinked tissue damage, even in the absence of hyperhomocysteinemia per se. An enzymatic component of high density lipoprotein, paraoxonase 1 (PON1), hydrolyses Hcy thiolactone, a metabolite of Hcy. Hcy thiolactone can add Hcy groups to (homocysteinylate) 
proteins thus damaging their structure and function [34]. It is therefore likely that mutations that reduce the ability of PON1 to hydrolyse and inactivate Hcy thiolactone will cause an increase in Hcy-mediated cell and tissue damage as this toxic metabolite accumulates, even without a concomitant increase in plasma Hcy.

Hyperhomocysteinemia has been observed in renal patients [35], as the kidney is responsible for $70 \%$ of peripheral Hcy metabolism [36]. Indeed as renal function declines with age, this contributes to the known age-related increase in Hcy [35]. A number of pharmacological reagents can influence Hcy levels. Methotextrate is a folate antagonist that is used to suppress cell proliferation in the treatment of both cancer and rheumatoid arthritis, and it is known that this agent increases plasma Hcy concentrations [37]. Conversely, estrogen-containing oral contraceptives reduce plasma Hcy levels [38], via upregulation of antioxidant genes such as glutathione peroxidase and $\mathrm{Mn}$-superoxide dismutase [39]. Due to the presence of endogenous estrogen, young women have lower Hcy levels than young men [40], and this sexual dimorphism is lost after the menopause [41]. Finally, lifestyle factors can influence Hcy levels with cigarette smoking, high levels of coffee consumption, and lack of exercise all known risk factors for elevated plasma Hcy [4].

\section{Evidence for Hcy-triggered Neurotoxicity}

Neurodegeneration is characterized by the death of neurons in specific regions of the nervous system leading to functional change. In an in vivo model of $\mathrm{PD}$, studies revealed that the proportion of murine midbrain dopaminergic neurons killed by the specific dopaminergic neurotoxin 1-methyl 4-phenyl 1,2,3,6-tetrahydropyridine (MPTP) was higher when plasma Hcy levels were elevated [42]. In vitro studies have shown direct toxicity of Hcy to a vast number of neuronal populations including cortical neurons [43], postnatal cerebellar granule neurons [44], cerebellar Purkinje neurons [45], dorsal root ganglion neurons [46], hippocampal neurons [47] and trigeminal sensory neurons [48]. Nonetheless the toxicity of Hcy to neurons is not universal with embryonic cerebellar granule neurons [44] and embryonic murine dopaminergic ventral mesencephalic neurons [49] both unaffected.

\section{Effects of Homocysteine on the NMDA Receptor}

\begin{tabular}{l|l|l}
$\begin{array}{l}\text { Homocysteine: } \\
\text { Low concentration }\end{array}$ & $\begin{array}{l}\text { Homocysteine at glutamate site = agonist } \\
\text { Homocysteine at glycine site = antagonist }\end{array}$ & $\begin{array}{l}\text { No effect on } \\
\text { cell survival }\end{array}$ \\
\hline $\begin{array}{l}\text { Homocysteine: } \\
\text { High concentration }\end{array}$ & $\begin{array}{l}\text { Homocysteine at glutamte site = AGONIST } \\
\text { Homocysteine at glycine site = antagonist }\end{array}$ & $\begin{array}{l}\text { Excitotoxicty and } \\
\text { cell death }\end{array}$ \\
\hline $\begin{array}{l}\text { Homocysteine: } \\
\text { Low concentration } \\
\begin{array}{l}\text { PLUS Glycine: } \\
\text { High concentration }\end{array}\end{array}$ & $\begin{array}{l}\text { Homocysteine at glutamate site = agonist } \\
\text { Glycine at glycine site = agonist }\end{array}$ & $\begin{array}{l}\text { Excitotoxicty and } \\
\text { cell death }\end{array}$ \\
& &
\end{tabular}

Figure 2: Actions of Homocysteine at the N-methyl-D-aspartate receptor Diagram summarising the actions of Hcy on the NMDA receptor. Hcy can act both as an agonist and an antagonist of this receptor by targeting different binding sites. The expression of other receptor agonists can influence the concentration of Hcy required to elicit excitotoxicity.
The signalling pathways that underpin Hcy-mediated neurotoxicity may vary with the neuronal population studied. Thus many investigations have focused on Hcy's interaction with the NMDA receptor (Figure 2). Hcy has agonistic effects on the glutamate binding site of the NMDA receptor, stimulating the receptor, and causing an excitotoxic response. However, Hcy is also a partial antagonist of the glycine coagonist site of the NMDA receptor, reducing glycine binding and therefore decreasing activation: an interaction that represses excitotoxicity. Thus it is believed that at low concentrations, equilibrium would be attained between Hcy's agonist and antagonist actions but at high concentrations the strong agonistic effect would prevail leading to cell death [50]. Evidence for a central role for the NMDA receptor in mediating Hcy-triggered neuronal death also arises from the finding that undifferentiated HT22 cells (a murine hippocampal neuronal cell line) are resistant to Hcy, whilst differentiated HT22 cells are not. The onset of susceptibility to Hcy corresponds with the onset of NMDA receptor expression, and as such antagonists of the NMDA receptor protect differentiated HT22 cells from Hcy-mediated apoptosis [51]. Excitotoxicity has also been reported following Hcy binding to the class I metabotropic glutamate receptors [52]. Accordingly, preventing Hcy binding to either the NMDA or class I metabotropic glutamate receptors via co-treatment with agonists of both these classes of receptors almost completely prevents Hcy-mediated cell death in postnatal cerebellar granule cells in vitro [53].

Hcy disrupts the DNA methylation cycle inducing DNA damage, cell death, and alterations in gene expression [54]. When Hcy levels rise, SAM levels decrease and SAH levels increase. This decreases the ratio of SAM to SAH, inducing DNA damage. Together with PARP activity, this triggers apoptosis in rat hippocampal neurons [47]. Elevated SAH expression also leads to DNA hypomethylation and alterations in gene expression that can trigger apoptosis and neuronal dysfunction [55]. Accordingly, SAM administration can reduce Hcymediated cortical neuron apoptosis [56] through re-balancing the ratio of SAM to SAH, and furthermore, SAM supplementation after ischemia improves neuronal viability in vivo [57].

In addition to its interactions with the NMDA receptor, Hcy can increase levels of cellular oxidative stress as it is a potent oxidising agent in its own right [58], and can regulate antioxidant enzymes [59]. A number of anti-oxidant agents have been shown to protect against cell death induced by Hcy. Carnosine, formed from the amino acids $\beta$-alanine and histadine, is a scavenger of reactive oxygen species (ROS). Thus it has been demonstrated that carnosine protects cerebellar granule neurons from Hcy toxicity by preventing the accumulation of ROS [60].

In contrast to studies on neuronal viability, far fewer investigations have considered the effects of Hcy on biochemistry, morphology, neurophysiology or neuronal function. It is known that Hcy inhibits neurite outgrowth from primary cultured dopaminergic neurons [49] and cerebellar Purkinje neurons [45]. This implies that Hcy can inhibit communication between neurons and the establishment of neuronal networks.

As outlined above, Hcy induces oxidative stress in neurons [59] and oxidative stress causes deficits in spatial learning and memory in aged rats. Thus it can be hypothesised that hyperhomocysteinemia will also cause deficits in spatial learning. Rats that have chronic hyperhomocysteinemia induced by methionine administration, exhibit spatial learning deficits and enhanced lipid peroxidation and glutathione reduction, which are biomarkers of cellular oxidative 
stress [61]. Melatonin is an antioxidant and reverses both the behavioural deficits and the incidence of the biochemical markers of oxidative stress. Likewise, the naturally-occurring anti-oxidant compound curcumin reduces both oxidative stress and cognitive impairment triggered by intracerebroventricular Hcy injection in rats [62]. These findings suggest that oxidative stress modulates the Hcymediated deficits in cognition and memory. Interestingly maternal hyperhomocysteinemia can have lasting consequences for neuronal function of the progeny. Thus sustained oxidative stress, coupled with a decrease in anti-oxidant enzyme expression, an increase in neuronal death, and impaired cognitive ability has been recorded in the offspring of rats with induced hyperhomocysteinemia, although the progeny do not have hyperhomocysteinemia themselves [63].

In addition to the well documented alterations in oxidative stress, Hcy can influence the expression of neurotransmitters and growth factors in hyperhomocysteinemic animals that exhibit cognitive deficits [64]. Thus reduced levels of dopamine and serotonin have been found in the cortex of such rats together with decreased levels of brain derived neurotrophic factor in the cerebrospinal fluid. Given the long established knowledge that these neurochemicals play fundamental roles in neuronal survival and function, further work evaluating how these contribute to Hcy-mediated alterations in neurophysiology is needed.

\section{Links between Hcy and Parkinson's Disease}

In a murine model of PD, experiments demonstrated that Hcy was not a dopaminergic neurotoxin in its own right. However, it markedly enhanced the neurotoxic effects of MPTP, increasing the magnitude of cell loss observed in the substantia nigra following exposure [42]. Thus Hcy potentiated the neurotoxic effects of other factors leading to the development of parkinsonian symptoms. Mixed data has been produced on whether or not Hcy per se is toxic to dopaminergic neurons in vitro, but again it enhanced the neurotoxic actions of MPTP in these cells $[49,65]$. Moreover, direct administration of Hcy intracerebroventricularly significantly decreases locomotor activity in rats, with a concomitant reduction in striatal dopamine levels [66]. These findings provide evidence that Hcy can be toxic to dopaminergic systems raising the possibility that Hcy is involved in the pathogenesis of PD.

Lewy bodies are neuronal cytoplasmic inclusions observed in PD neuropathology that contain protein aggregates including the protein a-synuclein [67]. It is known that Hcy can be converted within the cell to form Hcy thiolactone, which can trigger protein aggregation by incorporating Hcy residues into proteins, enhancing their propensity to form aggregates [34]. Thus a potential mechanism whereby Hcy could enhance $\alpha$-synuclein accumulation exists, although it remains to be verified. In addition, hypomethylation has been reported in the promoter region of $\alpha$-synuclein in the substantia nigra of Parkinson's patients to a greater degree than in other brain regions such as the anterior cingulate and putamen [68]. Hcy has been shown, as mentioned earlier, to induce DNA hypomethylation via lowering the SAM: SAH ratio. $\alpha$-synuclein itself can also indirectly decrease DNA methylation via its ability to associate with the DNA methylation enzyme Dnmt1, sequestering it out of the nucleus and into the cytoplasm thereby preventing it from methylating DNA [69]. Thus DNA hypomethylation could trigger overexpression of a-synuclein, which, in turn, would further reduce DNA methylation by sequestering Dnmt1. The importance of modulation of methylation pathways in PD has been further underlined by the discovery that adenosyl homocysteinase is differentially expressed in the Parkinson's as compared to the non-Parkinson's brain [70]. This enzyme converts SAH to Hcy and adenosine thus overexpression as seen in PD will lead to an increase in Hcy. Decreased methylation of the cytochrome P450 2E1 gene (CYP2E1) has been identified in PD patients [71]. Interestingly polymorphisms of this gene have already been associated with the development of PD in the Swedish population [72]. The enzymatic product of CYP2E1 co-localises with tyrosine hydroxylase in neurons of the rat substantia nigra [73] where it has been shown to modulate dopamine levels and to induce the production of ROS [74] suggesting a pathway whereby CYP2E1 can contribute to Parkinson's pathogenesis.

The role of the mitochondrion in the pathogenesis of neurodegenerative disorders, including $\mathrm{PD}$, has attracted much attention [75]. Electron microscopy has revealed that Hcy induces mitochondrial swelling, which is blocked by the addition of cyclosporin A that binds to the mitochondrial matrix protein, cyclophilin $\mathrm{D}$, preventing the calcium-dependent formation of the mitochondrial permeability transition ( $\mathrm{mPT}$ ) [53]. $\mathrm{mPT}$ formation is a critical step in the activation of cell death, linking Hcy-mediated mitochondrial damage to neuronal demise. Studies directly linking Hcy to mitochondrial dysfunction in neurons are limited. However, experiments on retinal ganglion cells from CBS-deficient mice demonstrate increased mitochondrial number in this tissue implying that mitochondrial-mediated generation of ROS could be increased [76]. Moreover, homocysteic acid, a metabolite of Hcy, induces deficiencies in mitochondrial complex I along with enhanced detection of markers of ROS-mediated damage in rat cerebral cortex [77]. Interestingly complex I deficiencies are strongly linked to PD demonstrating another potential mechanism whereby Hcy can modulate known pathways that lead to Parkinson's pathology.

Hcy can trigger neuroinflammation, and activated microglia and astrocytes release a number of factors that initiate an inflammatory response and that can trigger neuronal death. Neuroinflammation has been widely reported in the substantia nigra of PD patients [78] and attenuation of this has proved highly neuroprotective in PD models [79-81]. Of potential importance for the pathogenesis of $\mathrm{PD}$ is the release of NO by activated microglia and astrocytes [82]. NO has a number of deleterious effects on neurons that can lead to neurodegeneration, and its release has been detected following MPTP or 6-hydroxydopamine (6-OHDA) exposure in rodents [83] with co-administration of 6-OHDA and an NO scavenger preventing the neuronal death observed with 6-OHDA alone [84]. Furthermore NO can directly target proteins linked to PD by binding to them and S-nitrosylating them. Thus S-nitrosylation in PD models has been linked to both the erroneous activation of PKC- $\gamma$, leading to p53 activations and consequently neuronal death [85], and to the inactivation of neuroprotective proteins such as parkin [86], XIAP [87] and peroxiredoxin-2 [88], again leading to cell death. Inactivation or downregulation of these protective proteins has previously been linked to PD. In addition to Hcy initiating neuroinflammation, leading to NO release from the support cells of the nervous system, Hcy can trigger the release of NO within neurons themselves triggering apoptosis [48].

\section{Hcy and Parkinson's Risk and Progression}

A longitudinal study revealed that higher dietary intake of vitamin B6, but not folate or B12, was associated with a decreased risk of developing PD [89,90]. Vitamin B6 is an important co-factor in the pathways that mediate Hcy metabolism, however, the lack of correlation between dietary folate or B12 intake and PD suggests 
vitamin B6-mediated protection against PD is not directly related to Hcy levels. More recently it has been established that polymorphisms in key genes that mediate folate metabolism, including MHTFR, enhance the risk of developing PD in ethnic Chinese patients resident in Taiwan [91]. It has also been noted that the TT variant of the MTHFR C677T polymorphism is linked to an increased risk of PD in smokers [92]. These studies imply that Hcy plays a role in the pathogenesis of Parkinson's disease, with the possibility that Hcy is a risk factor remaining and further longitudinal studies on populations warranted.

It is well known that PD patients treated with levodopa (L-DOPA) have elevated plasma Hcy $[93,94]$. In vivo, the main metabolic fate of L-DOPA is O-methylation leading to the formation of 3-O-methyldopa, in a reaction catalysed by catechol-O-methyltransferase (COMT) with SAM acting as a methyl donor [95]. The donation of a methyl group from SAM leads to the formation of SAH, which in turn is hydrolysed to produce Hcy. This leads to high levels of Hcy in the plasma of L-DOPA-treated patients [93,96]. Crosssectional links observed between hyperhomocysteinemia and PD are likely to be largely caused by L-DOPA therapy rather than pre-existing hyperhomocysteinemia or hyperhomocysteinemia induced by PD per se. Elevated Hcy in response to L-DOPA can be counteracted through a program of exercise and thus in Parkinson's patients who do not exercise regularly a positive correlation is observed between L-DOPA dosage and serum Hcy. This correlation is not observed in Parkinson's patients who exercise regularly. Indeed patients in this latter group have serum Hcy concentrations that are indistinguishable from untreated control subjects [97]. Therefore lifestyle alterations could be used in suitable patients to counteract L-DOPA -mediated increases in Hcy. This may be of particular importance given that hyperhomocysteinemia has been identified as a risk factor for the occurrence of osteoporosis in PD patients [98]. Parkinson's patients have alterations in gait that coupled with their difficulty in motor control increases the likelihood of falls and fractures [99]. It has been demonstrated that $48 \%$ of Hcy found in bone exists bound to collagen in the extracellular bone matrix that might serve to weaken the bone enhancing the likelihood of fracture following a fall [100]. Thus reducing Hcy levels may prevent or reduce the severity of osteoporosis that would, in turn, reduce the probability of falls causing broken bones in these patients. Currently this has not been fully investigated but a small scale trial suggested that lowering Hcy levels using folate and B12 did indeed enhance bone density in Parkinson's patients [101]. However, it should be noted that all participants in this study were undergoing L-DOPA therapy and therefore the broader question of whether Hcy decreases bone density in all PD patients in the absence of L-DOPA remains to be determined.

The unified Parkinson's Disease rating scale (UPDRS) is a rating tool designed to assess disease burden in patients with Parkinson's Disease. The maximum score of 199 represents the worst (total) disability and 0 represents no disability. A positive correlation exists between Hcy levels and performance on the UPDRS in hyperhomocysteinemic patients [102]. However, as Hcy levels also correlate with L-DOPA dosage in this study further complicating the reasons for the link between Hcy and UPDRS score. The same study also noted a negative correlation between Hcy levels and levels of serum folate and B12. It has been demonstrated that administration of folate and vitamin B12 can lower plasma Hcy levels in L-DOPA-treated PD patients [103]. In this regard, it may be appropriate to determine whether folate and vitamin B12 supplementation in patients with hyperhomocysteinemia leads to a slowing of disease progression, as assessed by UPDRS.
Elevated Hcy has also been positively correlated to mild parkinsonian symptoms in the elderly, as assessed using an abbreviated motor portion of the UPDRS [104]. Thus Hcy maintains a complex relationship with PD. Whilst it is neurotoxic to dopaminergic neurons in experimental settings, there is mixed evidence on whether it is a risk factor for the development of PD. It is obvious, however, that Hcy has the potential to play a very important role in disease progression with hyperhomocysteinemia induced by L-DOPA that is widely used in the treatment of the disease and therefore it can be deduced that the likelihood of a PD patient developing hyperhomocysteinemia is high. Although it is clear that L-DOPA is a major contributor to hyperhomocysteinemia in PD patients, excess Hcy is well known to be deleterious to health [4]. The higher scoring on UPDRS in hyperhomocysteinemic patients, coupled with the increased risk of fracture following a fall highlights the potential importance of targeting Hcy levels in individuals diagnosed with PD.

\section{Conclusions and Discussion}

Taking an overarching view of the literature it is clear that Hcy has a number of deleterious effects that have significance for PD (Figure 3). Whilst it remains a matter for debate as to whether Hcy is a risk factor for PD development, the data linking elevated levels to an enhanced rate of disease progression are remarkably clear cut. Thus understanding the effects of Hcy on dopaminergic neurons is crucial in slowing down or halting this decline. It has been suggested that hyperhomocysteinemia can be caused by oxidative stress, rather than being causative of it. Thus a number of genetic alleles uncovered through studies of familial PD, such as parkin, PINK-1 and DJ-1 increase oxidative stress [105] independent of Hcy status. Under oxidative stress, methionine synthase is oxidatively labile, and levels of vitamin B12 and folate are depleted: biochemical changes that could trigger hyperhomocysteinemia [106]. It is known that in PD patients, treatment with L-DOPA causes hyperhomocysteinemia [93]. Therefore, although PD patient population have elevated levels of Hcy compared to controls in cross-sectional studies, it must be remembered that these can be triggered by both the oxidative stress observed in the disorder and by L-DOPA treatment.

Unlike Alzheimer's Disease where elevated Hcy levels are observed many years before the onset of symptoms giving a strong case for Hcy as a risk factor for the development of the disease or as a marker for early stage changes, there is no convincing data that this

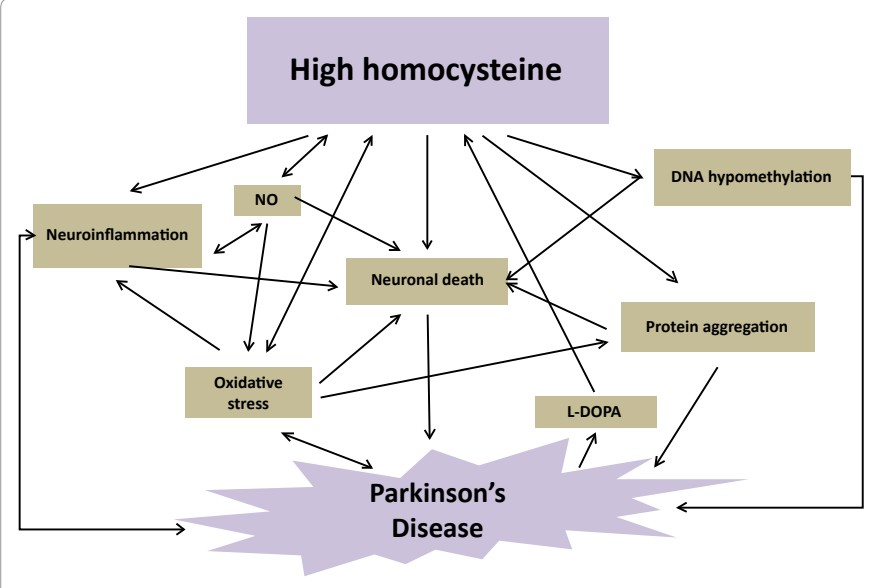

Figure 3: The complex relationship between Hcy and Parkinson's Disease Diagram demonstrating the main pathways linking Hcy to Parkinson's Disease and highlighting the complexity of this relationship. 
is the case for PD. Polymorphisms in the MTHFR gene can correlate with elevated plasma Hcy levels dependent on the folate status of the individual [28]. However, the proportion of individuals carrying the C677T MTHFR polymorphism is similar in PD-afflicted and control, age and gender-matched individuals [107,108]. Interestingly, patients carrying this polymorphism develop PD at a younger age [109]. This implies that these patients would develop PD anyway but that the rate of progression to symptomatic disease is escalated.

In recent years, research into the role of hypomethylation in epigenetic alterations linked to neurodegeneration in the elderly has expanded rapidly. Therefore, it is possible that changes in gene expression, brought about by elevated Hcy levels, are an early event in the pathogenesis of Hcy-linked neurodegenerative diseases. We could hypothesise that elevation of Hcy, as a risk factor for neurodegeneration, is an early event in the disease development, and that the changes in the expression of methylation-sensitive genes represent more distal steps in the linear progression of the disease. Under such circumstances, therapies targeting Hcy may be ineffective in stopping disease progression, and this may explain the failure of $B$ vitamin therapies to alter the course of neurodegeneration in the trials that have been reported to date. Thus perhaps Hcy-sensitive genes, rather than Hcy per se will prove more effective therapeutic targets for preventing PD progression in hyperhomocysteinemic individuals. Moreover, given that hyperhomocysteinemia leads to a decrease in SAM, it is plausible that strategies to specifically counteract Hcy-mediated hypomethylation more generally, rather than just targeting specific genes with aberrant expression will offer an exciting new drug development avenue in the search for agents to alleviate the progression and symptoms of neurodegeneration. In models of Alzheimer's disease administration of SAM delays the onset of expression of Alzheimer's biomarkers [110]. Likewise, SAM administration delays the onset of motor symptoms in amyotrophic lateral sclerosis (ALS) model mice [111]. Data for SAM administration in PD models are somewhat lacking. However, a small scale clinical study demonstrated beneficial effects of SAM administration in alleviating depressive symptoms in PD patients [112]. On a cautionary note, it must be mentioned that direct administration of SAM into the rat brain induces parkinsonian symptoms [113], most likely due to alterations in protein methylation and therefore SAM needs to be extensively tested and verified to ensure that it does not have deleterious effects with sustained or excessive use in PD models. L-DOPA administration can also decrease the SAM:SAH ratio leading to hypomethylation and dysfunction of cellular proteins in dopaminergic neurons in vitro, including the neuroprotective enzyme protein phosphatase 2A [114].

Another mechanism that is increasingly seen as being important in the pathogenesis of PD is neuroinflammation [82]. The microglia and astrocytes that make up the non-neuronal complement of the CNS serve a vast array of functions including nutritional and biochemical support for neurons, protection against oxidative stress and they mediate the CNS' immune defences. However, when activated to elicit an immune response in the CNS, the chemicals that they release to provide immunity can have maladaptive consequences for neuronal viability and function. Thus amongst the many mediators released by activated microglia and astrocytes in the immune response is NO. High levels of NO promote neuronal apoptosis and damage proteins S-nitrosylating them. Hcy can modulate NO signalling at a number of levels. Firstly Hcy has been shown to trigger activation of CNS immune cells thus the main site of production of NO in the CNS is switched on. Secondly, NO and its metabolite, peroxynitrite, have been shown to mediated Hcy induced cell death in neurons [48]. Furthermore, activation of the NMDA receptor leads to a rise in intracellular calcium and excessive nNOS activity and therefore generation of high levels of NO [115]. Thus it is plausible that intracellular rises in NO in response to Hcy are due to its known interactions with the NMDA receptor. In addition, NO can increase levels of Hcy. The 4a $4 \mathrm{~b}$ VNTR polymorphism in eNOS leads to enhanced NO production that, in turn, increases Hcy levels. Thus, a cycle could be established between $\mathrm{NO}$ and Hcy with both able to increase expression of each other and both negatively impacting on neurons.

The NMDA receptor seems to be intricately linked to Hcymediated cell loss in a number of circumstances. In PD patients, it has been proposed that antagonists of the NMDA receptor may alleviate dyskenesias associated with L-DOPA treatment and indeed clinical findings back up this hypothesis [116]. Antagonism of this receptor could theoretically reduce Hcy-mediated neurotoxicity (Figure 2) giving a potential added benefit of such drugs to L-DOPA-treated PD patients. It would be of great interest to test this hypothesis in PD models.

A number of pathways associated with Hcy-linked neuronal death and dysfunction converge on a central role for oxidative stress (Figure 3). Hyperhomocysteinemia can both induce and be induced by oxidative stress making it hard to tell which arose first. Although the temporal relationship between elevated Hcy and oxidative stress in PD is hard to ascertain, it is clear that, at least in experimental models, antioxidants can reduce the effects of Hcy. Given that, even in the absence of hyperhomocysteinemia, oxidative stress has been strongly linked to neuronal death in PD it is not surprising that a vast array of antioxidant compounds have proven beneficial in PD models including garcinia indica extract [117], gallic acid [118] and isothiocyanate erucin [119]. Whether these would have similar benefits in counteracting Hcy-mediated biochemical, cellular and behavioural change in PD pathways remains to be determined.

Reductions in plasma Hcy could have a number of benefits for Parkinson's patients. Most importantly given the positive correlation between Hcy levels and adverse performance on UPDRS, reduction of Hcy has the potential to slow down disease progression. Given the ease of lowering Hcy levels, for instance using exercise or B vitamins, it seems pertinent to use such strategies in patients and certainly this will do no harm. Unfortunately, it is unclear when the best point is to attempt to modify disease progress by lowering Hcy and therefore detailed longitudinal studies of patients treated at onset of symptoms, or at onset of L-DOPA therapy against those in which Hcy levels are not modified are warranted and required. Although PD is widely thought of as a movement disorder, depression and dementia can arise during the course of the disease. There is a positive correlation between hyperhomocysteinemia and dementia is PD patients in the majority of the small-scale studies carried out [120]. However, it should be noted that many progression to dementia tends to be a late event in the linear progression of the disease, many of the individuals who develop dementia will have been receiving L-DOPA for some time, and may well be on high doses of the drug which may be a confounding factor in this finding.

Given the irrefutable evidence that PD patients treated with L-DOPA have higher Hcy levels than control subjects, there is a paucity of studies detailing whether Hcy reduction strategies will slow down progression on UPDRS, onset of dementia or falls and fractures all of which occur with increased frequency in hyperhomocysteinemic PD patients. To ascertain the molecular pathways underpinning these 
events, further long term evaluation of L-DOPA treated PD model animals in which hyperhomocysteinemia has been attenuated against those in which it has not been, is essential, together with further analysis of clinical data. A recent publication opined that the future for PD therapies would combine a number of features including the ability to reduce oxidative stress and Hcy whilst enhancing the bioavailability of methyl groups [121]. Whilst there is no clear cut data showing that Hcy lowering therapies will provide long term benefits to PD patients, the weight of evidence suggests that it would not be harmful to keep Hcy levels in check in PD patients and this practice should perhaps be more widespread.

\section{Acknowledgements}

GHD holds an Alzheimer's Society personal research fellowship (grant number 93) with support from the Henry Smith Charity. Alzheimer's Society is a charity (registration no. 296645) and a company registered in England and Wales (registration no. 2115499). The University of St Andrews is a charity registered in Scotland (registration no. SC013532).

\section{References}

1. Tchantchou $F$ (2006) Homocysteine metabolism and various consequences of folate deficiency. J Alzheimers Dis 9: 421-427.

2. Porto MP, Galdieri LC, Pereira VG, Vergani N, da Rocha JC, et al. (2005) Molecular analysis of homocystinuria in Brazilian patients. Clin Chim Acta 362: 71-78.

3. de Lau LM, Breteler MM (2006) Epidemiology of Parkinson's disease. Lancet Neurol 5: 525-535

4. Doherty GH (2008) Boom and Bust for Homocysteine? Central Nervous System Agents in Medicinal Chemistry 8: 107-120.

5. Thomas B, Beal MF (2007) Parkinson's disease. Hum Mol Genet.

6. Reynolds A, Laurie C, Mosley RL, Gendelman HE (2007) Oxidative stress and the pathogenesis of neurodegenerative disorders. Int Rev Neurobiol 82 : 297-325.

7. Franco OH, Kirkwood TB, Powell JR, Catt M, Goodwin J, et al. (2007) Ten commandments for the future of ageing research in the UK: a vision for action. BMC Geriatr 7: 10.

8. Cutler RG, Mattson MP (2006) The adversities of aging. Ageing Res Rev 5: 221-238.

9. KostiÄ $\ddagger$ VS (2009) Treatment of young-onset Parkinson's disease: role of dopamine receptor agonists. Parkinsonism Relat Disord 15: S71-S75.

10. Collier TJ, Kanaan NM, Kordower JH (2011) Ageing as a primary risk factor for Parkinson's disease: evidence from studies of non-human primates. Nat Rev Neurosci 12: 359-366.

11. Allain P, Le Bouil A, Cordillet E, Le Quay L, Bagheri H, et al. (1995) Sulfate and cysteine levels in the plasma of patients with Parkinson's disease. Neurotoxicology 16: 527-529.

12. Keller EB, Rachele JR, DU Vigneaud V (1949) A study of transmethylation with methionine containing deuterium and $\mathrm{C} 14$ in the methyl group. J Biol Chem 177: 733-738.

13. CAntoni GL, Vignos PJ Jr (1954) Enzymatic mechanism of creatine synthesis. J Biol Chem 209: 647-659.

14. Zhang L, Lee HK, Pruess TH, White HS, Bulaj G (2009) Synthesis and applications of polyamine amino acid residues: improving the bioactivity of an analgesic neuropeptide, neurotensin. J Med Chem 52: 1514-1517.

15. Berger ML, Pöhler T, Schadt O, Stanger M, Rebernik P, et al. (2013) Exploring the polyamine regulatory site of the NMDA receptor: a parallel synthesis approach. ChemMedChem 8: 82-94.

16. Booker SJ, Grove TL (2010) Mechanistic and functional versatility of radical SAM enzymes. F1000 Biol Rep 2: 52.

17. DE LA HABA G, CANTONI GL (1959) The enzymatic synthesis of S-adenosylL-homocysteine from adenosine and homocysteine. J Biol Chem 234: 603608.

18. Skiba WE, Taylor MP, Wells MS, Mangum JH, Awad WM Jr. (1982) Human hepatic methionine biosynthesis. Purification and characterization of betaine:Hcy S-methyltransferase. J Biol Chem 257: 14944-14948.

19. Loughlin RE, Elford HL, Buchanan JM (1964) Enzymatic synthesis of the methyl group of methionine. Vii. Isolation of a cobalamin-containing transmethylase (5-methyltetrahydro-folate-homocysteine) from mammalian liver. J Biol Chem 239: 2888-2895.

20. Mudd SH, Finkelstein JD, Irreverre F, Laster L (1965) Transsulfuration in mammals. Microassays and tissue distributions of three enzymes of the pathway. J Biol Chem 240: 4382-4392.

21. Flavin M, Segal A (1964) Purification and properties of the cystathionine gamma-cleavage enzyme of neurospora. J Biol Chem 239: 2220-2227.

22. Rassin DK, Sturman JA (1975) Cysteine sulfinic acid decarboxylase in rat brain: effect of vitamin B6 deficiency on soluble and particulate components. Life Sci 16: 875-881.

23. Finkelstein JD (1990) Methionine metabolism in mammals. J Nutr Biochem 1: 228-237.

24. Vitvitsky V, Thomas M, Ghorpade A, Gendelman HE, Banerjee R (2006) A functional transsulfuration pathway in the brain links to glutathione homeostasis. J Biol Chem 281: 35785-35793.

25. Harpey JP, Rosenblatt DS, Cooper BA, Le Moël G, Roy C, et al. (1981) Homocystinuria caused by 5,10-methylenetetrahydrofolate reductase deficiency: a case in an infant responding to methionine, folinic acid, pyridoxine, and vitamin B12 therapy. J Pediatr 98: 275-278.

26. HaanEA, Rogers JG, LewisGP,RowePB(1985)5,10-Methylenetetrahydrofolate reductase deficiency. Clinical and biochemical features of a further case. $J$ Inherit Metab Dis 8: 53-57.

27. Elsaid MF, Bener A, Lindner M, Alzyoud M, Shahbek N, et al. (2007) Are heterocygotes for classical homocystinuria at risk of vitamin B12 and folic acid deficiency? Mol Genet Metab 92: 100-103.

28. Jacques PF, Bostom AG, Williams RR, Ellison RC, Eckfeldt JH, et al. (1996) Relation between folate status, a common mutation in methylenetetrahydrofolate reductase, and plasma homocysteine concentrations. Circulation 93: 7-9.

29. Jemaa R, Kallel A, Sediri Y, Abdessalem S, Mourali MS, et al. (2012) Association between endothelial nitric oxide gene intron 4a4b VNTR polymorphism and plasma homocysteine concentrations in Tunisian male patients with myocardial infarction. Nutr Res 32: 342-346.

30. Wang XL, Mahaney MC, Sim AS, Wang J, Wang J, et al. (1997) Genetic contribution of the endothelial constitutive nitric oxide synthase gene to plasma nitric oxide levels. Arterioscler Thromb Vasc Biol 17: 3147-3153.

31. Danishpajooh IO, Gudi T, Chen Y, Kharitonov VG, Sharma VS, et al. (2001) Nitric oxide inhibits methionine synthase activity in vivo and disrupts carbon flow through the folate pathway. J Biol Chem 276: 27296-27303.

32. Juckett MB, Weber M, Balla J, Jacob HS, Vercellotti GM (1996) Nitric oxide donors modulate ferritin and protect endothelium from oxidative injury. Free Radic Biol Med 20: 63-73.

33. Suh JR, Oppenheim EW, Girgis S, Stover PJ (2000) Purification and properties of a folate-catabolizing enzyme. J Biol Chem 275: 35646-35655.

34. Jakubowski H (2000) Calcium-dependent human serum homocysteine thiolactone hydrolase. A protective mechanism against protein N-homocysteinylation. J Biol Chem 275: 3957-3962.

35. Garibotto G, Sofia A, Valli A, Tarroni A, Di Martino M, et al. (2006) Causes of hyperhomocysteinemia in patients with chronic kidney diseases. Semin Nephrol 26: 3-7.

36. McKinley MC (2000) Nutritional aspects and possible pathological mechanisms of hyperhomocysteinaemia: an independent risk factor for vascular disease. Proc Nutr Soc 59: 221-237.

37. Refsum H, Ueland PM (1990) Clinical significance of pharmacologica modulation of homocysteine metabolism. Trends Pharmacol Sci 11: 411-416.

38. Brattström L, Israelsson B, Olsson A, Andersson A, Hultberg B (1992) Plasma homocysteine in women on oral oestrogen-containing contraceptives and in men with oestrogen-treated prostatic carcinoma. Scand J Clin Lab Invest 52: 283-287.

39. Viña J, Sastre J, Pallardó FV, Gambini J, Borrás C (2008) Modulation of 
longevity-associated genes by estrogens or phytoestrogens. Biol Chem 389: 273-277.

40. Boers GH, Smals AG, Trijbels FJ, Leermakers Al, Kloppenborg PW (1983) Unique efficiency of methionine metabolism in premenopausal women may protect against vascular disease in the reproductive years. J Clin Invest 72 : 1971-1976.

41. Verhoef P, Meleady R, Daly LE, Graham IM, Robinson K, et al. (1999) Homocysteine, vitamin status and risk of vascular disease; effects of gender and menopausal status. European COMAC Group. Eur Heart J 20: 1234 1244.

42. Duan W, Ladenheim B, Cutler RG, Kruman II, Cadet JL, et al. (2002) Dietary folate deficiency and elevated homocysteine levels endanger dopaminergic neurons in models of Parkinson's disease. J Neurochem 80: 101-110.

43. White AR, Huang X, Jobling MF, Barrow CJ, Beyreuther K, et al. (2001) Homocysteine potentiates copper- and amyloid beta peptide-mediated toxicity in primary neuronal cultures: possible risk factors in the Alzheimer'stype neurodegenerative pathways. J Neurochem 76: 1509-1520.

44. Foister NS, Oldreive CE, Mackie JB, Doherty GH (2005) Embryonic cerebellar granule cells are resistant to necrosis induced by homocysteine. Brain Res Dev Brain Res 160: 85-89.

45. Oldreive CE, Doherty GH (2007) Neurotoxic effects of homocysteine on cerebellar Purkinje neurons in vitro. Neurosci Lett 413: 52-57.

46. Tjiattas L, Ortiz DO, Dhivant S, Mitton K, Rogers E, et al. (2004) Folate deficiency and homocysteine induce toxicity in cultured dorsal root ganglion neurons via cytosolic calcium accumulation. Aging Cell 3: 71-76.

47. Kruman II, Kumaravel TS, Lohani A, Pedersen WA, Cutler RG, et al. (2002) Folic acid deficiency and homocysteine impair DNA repair in hippocampal neurons and sensitize them to amyloid toxicity in experimental models of Alzheimer's disease. J Neurosci 22: 1752-1762.

48. Williams HM, Lippok H, Doherty GH (2008) Nitric oxide and peroxynitrite signalling triggers homocysteine-mediated apoptosis in trigeminal sensory neurons in vitro. Neurosci Res 60: 380-388.

49. Heider I, Lehmensiek V, Lenk T, Müller T, Storch A (2004) Dopaminergic neurotoxicity of homocysteine and its derivatives in primary mesencephalic cultures. J Neural Transm 1-13.

50. Lipton SA, Kim WK, Choi YB, Kumar S, D’Emilia DM, et al. (1997) Neurotoxicity associated with dual actions of homocysteine at the N-methyl-D-aspartate receptor. Proc Natl Acad Sci U S A 94: 5923-5928.

51. Zhao Z, Lu R, Zhang B, Shen J, Yang L, et al. (2012) Differentiation of HT22 neurons induces expression of NMDA receptor that mediates homocysteine cytotoxicity. Neurol Res 34: 38-43.

52. Zieminska E, Stafiej A, Lazarewicz JW (2003) Role of group I metabotropic glutamate receptors and NMDA receptors in homocysteine-evoked acute neurodegeneration of cultured cerebellar granule neurones. Neurochem Int 43: 481-492

53. Zieminska E, Lazarewicz JW (2006) Excitotoxic neuronal injury in chronic homocysteine neurotoxicity studied in vitro: the role of NMDA and group metabotropic glutamate receptors. Acta Neurobiol Exp (Wars) 66: 301-309.

54. Wainfan E, Poirier LA (1992) Methyl groups in carcinogenesis: effects on DNA methylation and gene expression. Cancer Res 52: 2071s-2077s.

55. Obeid R, Herrmann W (2006) Mechanisms of homocysteine neurotoxicity in neurodegenerative diseases with special reference to dementia. FEBS Lett 580: 2994-3005.

56. Ho PI, Ortiz D, Rogers E, Shea TB (2002) Multiple aspects of homocysteine neurotoxicity: glutamate excitotoxicity, kinase hyperactivation and DNA damage. J Neurosci Res 70: 694-702.

57. Rao AM, Baskaya MK, Maley ME, Kindy MS, Dempsey RJ (1997) Beneficial effects of S-adenosyl-L-methionine on blood-brain barrier breakdown and neuronal survival after transient cerebral ischemia in gerbils. Brain Res Mol Brain Res 44: 134-138

58. Doherty GH (2007) Homocysteine: more than just a matter of life and death. Exp Neurol 205: 5-8.

59. Handy DE, Zhang Y, Loscalzo J (2005) Homocysteine down-regulates cellular glutathione peroxidase (GPx1) by decreasing translation. J Biol Chem 280: $15518-15525$
60. Kulebyakin K, Karpova L, Lakonsteva E, Krasavin M, Boldyrev A (2012) Carnosine protects neurons against oxidative stress and modulates the time profile of MAPK cascade signaling. Amino Acids 43: 91-96.

61. Baydas G, Ozer M, Yasar A, Tuzcu M, Koz ST (2005) Melatonin improves learning and memory performances impaired by hyperhomocysteinemia in rats. Brain Res 1046: 187-194.

62. Ataie A, Sabetkasaei M, Haghparast A, Moghaddam AH, Ataee R, et al (2010) Curcumin exerts neuroprotective effects against homocysteine intracerebroventricular injection-induced cognitive impairment and oxidative stress in rat brain. J Med Food 13: 821-826

63. Makhro AV, Mashkina AP, Solenaya OA, Trunova OA, Kozina LS, et al. (2008) Prenatal hyperhomocysteinemia as a model of oxidative stress of the brain. Bull Exp Biol Med 146: 33-35.

64. Gao L, Zeng XN, Guo HM, Wu XM, Chen HJ, et al. (2012) Cognitive and neurochemical alterations in hyperhomocysteinemic rat. Neurol Sci 33: 39 43.

65. Imamura K, Takeshima T, Nakaso K, Nakashima K (2007) Homocysteine is toxic for dopaminergic neurons in primary mesencephalic culture. Neuroreport 18: 1319-1322.

66. Lee ES, Chen H, Soliman KF, Charlton CG (2005) Effects of homocysteine on the dopaminergic system and behavior in rodents. Neurotoxicology 26: 361-371.

67. Kawahara K, Hashimoto M, Bar-On P, Ho GJ, Crews L, et al. (2008) alphaSynuclein aggregates interfere with Parkin solubility and distribution: role in the pathogenesis of Parkinson disease. J Biol Chem 283: 6979-6987.

68. Matsumoto L, Takuma H, Tamaoka A, Kurisaki H, Date H, et al. (2010) CpG demethylation enhances alpha-synuclein expression and affects the pathogenesis of Parkinson's disease. PLoS One 5: e15522.

69. Desplats P, Spencer B, Coffee E, Patel P, Michael S, et al. (2011) Alphasynuclein sequesters Dnmt1 from the nucleus: a novel mechanism for epigenetic alterations in Lewy body diseases. J Biol Chem 286: 9031-9037.

70. Werner CJ, Heyny-von Haussen R, Mall G, Wolf S (2008) Proteome analysis of human substantia nigra in Parkinson's disease. Proteome Sci 6: 8.

71. Kaut O, Schmitt I, Wüllner U (2012) Genome-scale methylation analysis of Parkinson's disease patients' brains reveals DNA hypomethylation and increased mRNA expression of cytochrome P450 2E1. Neurogenetics 13: 87-91.

72. Shahabi HN, Westberg L, Melke J, Håkansson A, Belin AC, et al. (2009) Cytochrome P450 2E1 gene polymorphisms/haplotypes and Parkinson's disease in a Swedish population. J Neural Transm 116: 567-573.

73. Watts PM, Riedl AG, Douek DC, Edwards RJ, Boobis AR, et al. (1998) Co-localization of P450 enzymes in the rat substantia nigra with tyrosine hydroxylase. Neuroscience 86: 511-519.

74. Shahabi HN, Andersson DR, Nissbrandt H (2008) Cytochrome P450 2 E1 in the substantia nigra: relevance for dopaminergic neurotransmission and free radical production. Synapse 62: 379-388.

75. Chin MH, Qian WJ, Wang H, Petyuk VA, Bloom JS, et al. (2008) Mitochondrial dysfunction, oxidative stress, and apoptosis revealed by proteomic and transcriptomic analyses of the striata in two mouse models of Parkinson's disease. J Proteome Res 7: 666-677.

76. Ganapathy PS, Perry RL, Tawfik A, Smith RM, Perry E, et al. (2011) Homocysteine-mediated modulation of mitochondrial dynamics in retinal ganglion cells. Invest Ophthalmol Vis Sci 52: 5551-5558.

77. Folbergrová J, Jesina P, Haugvicová R, Lisý V, Houstek J (2010) Sustained deficiency of mitochondrial complex I activity during long periods of survival after seizures induced in immature rats by homocysteic acid. Neurochem Int 56: 394-403.

78. Kohutnicka M, Lewandowska E, Kurkowska-Jastrzebska I, CzÅ onkowski A $\mathrm{C} z \AA$, onkowska A (1998) Microglial and astrocytic involvement in a murine model of Parkinson's disease induced by 1-methyl-4-phenyl-1,2,3,6tetrahydropyridine (MPTP). Immunopharmacology 39: 167-180.

79. Wu DC, Tieu K, Cohen O, Choi DK, Vila M, et al. (2002) Glial cell response: A pathogenic factor in Parkinson's disease. J Neurovirol 8: 551-558.

80. Choi DK, Pennathur S, Perier C, Tieu K, Teismann P, et al. (2005) Ablation of 
the inflammatory enzyme myeloperoxidase mitigates features of Parkinson's disease in mice. J Neurosci 25: 6594-6600.

81. Vijitruth R, Liu M, Choi DY, Nguyen XV, Hunter RL, et al. (2006) Cyclooxygenase-2 mediates microglial activation and secondary dopaminergic cell death in the mouse MPTP model of Parkinson's disease. J Neuroinflammation 3: 6 .

82. Doherty GH (2011) Nitric oxide in neurodegeneration: potential benefits of non-steroidal anti-inflammatories. Neurosci Bull 27: 366-382.

83. Liberatore GT, Jackson-Lewis V, Vukosavic S, Mandir AS, Vila M, et al. (1999) Inducible nitric oxide synthase stimulates dopaminergic neurodegeneration in the MPTP model of Parkinson disease. Nat Med 5: 1403-1409.

84. Pierucci M, Di Matteo V, Benigno A, Crescimanno G, Esposito E, et al. (2009) The unilateral nigral lesion induces dramatic bilateral modification on rat brain monoamine neurochemistry. Ann N Y Acad Sci 1155: 316-323.

85. Lee SJ, Kim DC, Choi BH, Ha H, Kim KT (2006) Regulation of p53 by activated protein kinase $\mathrm{C}$-delta during nitric oxide-induced dopaminergic cell death. J Biol Chem 281: 2215-2224.

86. Chung KK, Thomas B, Li X, Pletnikova O, Troncoso JC, et al. (2004) S-nitrosylation of parkin regulates ubiquitination and compromises parkin's protective function. Science 304: 1328-1331.

87. Tsang AH, Lee YI, Ko HS, Savitt JM, Pletnikova O, et al. (2009) S-nitrosylation of XIAP compromises neuronal survival in Parkinson's disease. Proc Natl Acad Sci U S A 106: 4900-4905.

88. Fang J, Nakamura T, Cho DH, Gu Z, Lipton SA (2007) S-nitrosylation of peroxiredoxin 2 promotes oxidative stress-induced neuronal cell death in Parkinson's disease. Proc Natl Acad Sci U S A 104: 18742-18747.

89. de Lau LM, Koudstaal PJ, Witteman JC, Hofman A, Breteler MM (2006) Dietary folate, vitamin B12, and vitamin B6 and the risk of Parkinson disease. Neurology 67: 315-318.

90. Murakami K, Miyake Y, Sasaki S, Tanaka K, Fukushima W, et al. (2010) Dietary intake of folate, vitamin B6, vitamin B12 and riboflavin and risk of Parkinson's disease: a case-control study in Japan. Br J Nutr 104: 757-764.

91. Fong CS, Shyu HY, Shieh JC, Fu YP, Chin TY, et al. (2011) Association of MTHFR, MTR, and MTRR polymorphisms with Parkinson's disease among ethnic Chinese in Taiwan. Clin Chim Acta 412: 332-338.

92. de Lau LM, Koudstaal PJ, van Meurs JB, Uitterlinden AG, Hofman A, et al (2005) Methylenetetrahydrofolate reductase C677T genotype and PD. Ann Neurol 57: 927-930.

93. Allain P, Le Bouil A, Cordillet E, Le Quay L, Bagheri H, et al. (1995) Sulfate and cysteine levels in the plasma of patients with Parkinson's disease. Neurotoxicology 16: 527-529.

94. Yasui K, Nakaso K, Kowa H, Takeshima T, Nakashima K (2003) Levodopainduced hyperhomocysteinaemia in Parkinson's disease. Acta Neurol Scand 108: $66-67$.

95. Cheng H, Gomes-Trolin C, Aquilonius SM, Steinberg A, Löfberg C, et al. (1997) Levels of L-methionine S-adenosyltransferase activity in erythrocytes and concentrations of S-adenosylmethionine and S-adenosylhomocysteine in whole blood of patients with Parkinson's disease. Exp Neurol 145: 580 585

96. Chandra G, Gangopadhyay PK, Senthil Kumar KS, Mohanakumar KP (2006) Acute intranigral homocysteine administration produces stereotypic behavioral changes and striatal dopamine depletion in Sprague-Dawley rats. Brain Res 1075: 81-92.

97. Nascimento CM, Stella F, Garlipp CR, Santos RF, Gobbi S, et al. (2011) Serum homocysteine and physical exercise in patients with Parkinson's disease. Psychogeriatrics 11: 105-112.

98. Lee SH, Kim MJ, Kim BJ, Kim SR, Chun S, et al. (2010) Hyperhomocysteinemia due to levodopa treatment as a risk factor for osteoporosis in patients with Parkinson's disease. Calcif Tissue Int 86: 132-141.

99. Johnell O, Melton LJ $3^{\text {rd }}$, Atkinson EJ, O'Fallon WM, Kurland LT (1992) Fracture risk in patients with parkinsonism: a population-based study in Olmsted County, Minnesota. Age Ageing 21: 32-38.

100. Holstein JH, Herrmann M, Splett C, Herrmann W, Garcia P, et al. (2011) High bone concentrations of homocysteine are associated with altered bone morphology in humans. Br J Nutr 106: 378-382.
101. Lee SH, Kim MJ, Kim BJ, Kim SR, Chun S, et al. (2010) Homocysteinelowering therapy or antioxidant therapy for bone loss in Parkinson's disease. Mov Disord 25: 332-340.

102. Ozer F, Meral H, Hanoglu L, Aydemir T, Yilsen M, et al. (2006) Plasma homocysteine levels in patients treated with levodopa: motor and cognitive associations. Neurol Res 28: 853-858.

103. Postuma RB, Espay AJ, Zadikoff C, Suchowersky O, Martin WR, et al. (2006) Vitamins and entacapone in levodopa-induced hyperhomocysteinemia: a randomized controlled study. Neurology 66: 1941-1943.

104.Louis ED, Schupf N, Tang MX, Marder K, Luchsinger JA (2007) Mild parkinsonian signs and plasma homocysteine concentration in communitydwelling elderly individuals. Arch Neurol 64: 1646-1651.

105. Nunomura A, Moreira PI, Lee HG, Zhu X, Castellani RJ, et al. (2007) Neuronal death and survival under oxidative stress in Alzheimer and Parkinson diseases. CNS Neurol Disord Drug Targets 6: 411-423.

106. McCaddon A, Regland B, Hudson P, Davies G (2002) Functional vitamin $\mathrm{B}(12)$ deficiency and Alzheimer disease. Neurology 58: 1395-1399.

107. Religa D, Czyzewski K, Styczynska M, Peplonska B, Lokk J, et al. (2006) Hyperhomocysteinemia and methylenetetrahydrofolate reductase polymorphism in patients with Parkinson's disease. Neurosci Lett 404: 56 60.

108. Caccamo D, Gorgone G, Currò M, Parisi G, Di lorio W, et al. (2007) Effect of MTHFR polymorphisms on hyperhomocysteinemia in levodopa-treated Parkinsonian patients. Neuromolecular Med 9: 249-254.

109. Lin JJ, Yueh KC, Liu CS, Liu JT, Lin SZ (2007) 5,10-methylenetetrahydrofolate reductase $\mathrm{C677T}$ gene polymorphism can influence age at onset of Parkinson's disease. Acta Neurol Taiwan 16: 150-157.

110. Lee S, Lemere CA, Frost JL, Shea TB (2012) Dietary supplementation with S-adenosyl methionine delayed amyloid- $\hat{I}^{2}$ and tau pathology in $3 \times \mathrm{Tg}-\mathrm{AD}$ mice. J Alzheimers Dis 28: 423-431.

111. Suchy J, Lee S, Ahmed A, Shea TB (2010) Dietary supplementation with S-adenosyl methionine delays the onset of motor neuron pathology in a murine model of amyotrophic lateral sclerosis. Neuromolecular Med 12: 86 97.

112. Di Rocco A, Rogers JD, Brown R, Werner P, Bottiglieri T (2000) S-AdenosylMethionine improves depression in patients with Parkinson's disease in an open-label clinical trial. Mov Disord 15: 1225-1229.

113. Lamango NS, Charlton CG (2000) Farnesyl-L-cysteine analogs block SAMinduced Parkinson's disease-like symptoms in rats. Pharmacol Biochem Behav 66: 841-849.

114. Bottiglieri T, Arning E, Wasek B, Nunbhakdi-Craig V, Sontag JM, et al. (2012) Acute administration of L-DOPA induces changes in methylation metabolites, reduced protein phosphatase $2 \mathrm{~A}$ methylation, and hyperphosphorylation of Tau protein in mouse brain. J Neurosci 32: 9173-9181.

115. Dawson VL, Dawson TM, London ED, Bredt DS, Snyder SH (1991) Nitric oxide mediates glutamate neurotoxicity in primary cortical cultures. Proc Natl Acad Sci U S A 88: 6368-6371.

116. Elahi B, Phielipp N, Chen R (2012) N-Methyl-D-Aspartate antagonists in levodopa induced dyskinesia: a meta-analysis. Can J Neurol Sci 39: 465472.

117. Antala BV, Patel MS, Bhuva SV, Gupta S, Rabadiya S, et al. (2012) Protective effect of methanolic extract of Garcinia indica fruits in 6-OHDA rat model of Parkinson's disease. Indian J Pharmacol 44: 683-687.

118. Mansouri MT, Farbood Y, Sameri MJ, Sarkaki A, Naghizadeh B, et al. (2013) Neuroprotective effects of oral gallic acid against oxidative stress induced by 6-hydroxydopamine in rats. Food Chem 138: 1028-1033

119. Tarozzi A, Morroni F, Bolondi C, Sita G, Hrelia P, et al. (2012) Neuroprotective Effects of Erucin against 6-Hydroxydopamine-Induced Oxidative Damage in a Dopaminergic-like Neuroblastoma Cell Line. Int J Mol Sci 13: 1089910910.

120.Zoccolella S, Lamberti SV, lliceto G, Santamato A, Lamberti P, et al. (2010) Hyperhomocysteinemia in L-dopa treated patients with Parkinson's disease: potential implications in cognitive dysfunction and dementia? Curr Med Chem 17: 3253-3261.

121. Müller T (2011) Motor complications, levodopa metabolism and progression of Parkinson's disease. Expert Opin Drug Metab Toxicol 7: 847-855. 